\title{
Local Accumulation of Acetylcholine Receptors Is Neither Necessary Nor Sufficient to Induce Cluster Formation
}

\author{
Jes Stollberg and Scott E. Fraser \\ Department of Physiology and Biophysics, College of Medicine, University of California, Irvine, Irvine, California 92717
}

\begin{abstract}
Acetylcholine receptors (AChRs) accumulate at developing neuromuscular junctions in part via lateral migration of diffusely expressed receptors. Using a model system-cultured Xenopus muscle cells exposed to electric fields-we have shown that AChRs, concentrated at the cathode-facing cell pole, continue to aggregate there after the field is terminated (Stollberg and Fraser, 1988). These observations are consistent with the possibility that the field-induced increase in receptor concentration triggers the aggregation event. Only 2 other molecular events could initiate the electric field-induced receptor aggregation: (1) a local increase in the density of some other molecules, or (2) a voltage-
\end{abstract} sensitive mechanism.

Treatment of muscle cell cultures with neuraminidase changes the cell surface charge and has been reported to reverse the direction of electromigration for $\mathrm{AChRs}$ and concanavalin A binding sites (Orida and Poo, 1978). Using digitally analyzed fluorescence videomicroscopy, we find that AChRs in neuraminidase-treated cultures accumulate at both cell poles in an electric field. After termination of the field, the AChR continues to aggregate at the cathode-facing pole, as in cells not treated with neuraminidase. However, receptor density decreases at the anode-facing pole, indicating that elevated AChR density does not initiate receptor aggregation. Cells pretreated with neuraminidase and trypsin (which blocks receptor aggregation) display reversed receptor distributions compared to untreated controls, indicating that electromigration has indeed been reversed. The rate at which neuraminidase- and trypsin-treated cells approach steadystate distributions indicates a receptor diffusion constant of $\approx 1.2 \times 10^{-9} \mathrm{~cm}^{2} / \mathrm{sec}$, consistent with a diffusion trap mechanism of receptor aggregation.

These results are the first conclusive demonstration that the local concentration of receptors is neither necessary nor sufficient to induce receptor clustering. Our observations suggest that receptor clustering is triggered by the accumulation of some other molecules, or by a voltage-sensitive mechanism.

An important problem in developmental neurobiology concerns the localization of specific molecules at synapses. A much studied example is the neuromuscular junction, at which acetylcho-

\footnotetext{
Received May 3, 1989; revised July 21, 1989; accepted July 25, 1989.

This work was supported by a grant from the Monsanto Corporation and by N.I.H. Training Grant HD07029.

Correspondence should be addressed to Jes Stollberg at the above address.

Copyright (C) 1990 Society for Neuroscience 0270-6474/90/010247-09\$02.00/0
}

line receptors (AChRs), acetylcholinesterase, basal lamina components, and numerous cytoskeletal elements are concentrated (for review see Dennis, 1981; Schuetze and Role, 1987). Prior to innervation, the AChRs are distributed diffusely; clustering begins shortly after neuronal contact both in vivo (Blackshaw and Warner, 1976; Creazzo and Sohal, 1983) and in vitro (Frank and Fischbach, 1979; Kidokoro et al., 1980; Role et al., 1987). The development and stabilization of receptor clusters continues over a period of days to weeks and is likely to involve multiple mechanisms acting in concert as the cluster matures. It is of crucial importance, therefore, to consider the time frame of experiments directed at questions of mechanism. The focus of this report is on the initial events (i.e., within the first few minutes) responsible for triggering receptor clustering.

Experiments following the distribution of labeled AChRs indicate that lateral migration of diffusely expressed receptors makes a significant contribution to the initial clustering event (Anderson and Cohen, 1977; Frank and Fischbach, 1979; Kuromi and Kidokoro, 1984; Ziskind-Conhaim et al., 1984; Role et al., 1985; Stollberg and Fraser, 1988). In Xenopus, this migration has been shown to be consistent with the action of a diffusion trap mechanism (Edwards and Frisch, 1976), in which passively diffusing receptors are locally immobilized (Kuromi and Kidokoro, 1984; Stollberg and Fraser, 1988). The selective trapping of $A C h R s$ and other synaptic components is presumably mediated by binding to membrane components, to extracellular matrix molecules, or to cytoskeletal elements. The initial events that trigger the relevant interactions remain to be elucidated.

The clustering of membrane components can be studied by monitoring their redistribution on cultured cells in response to externally applied electric fields (Jaffe, 1977; Poo and Robinson, 1977; Poo et al., 1978; Luther and Peng, 1985). The technique permits an experimental manipulation of molecular distributions that is independent of exogenous factors and cell contacts, thereby facilitating quantitative analyses of receptor clustering. This approach has been used to particular advantage with spherical muscle cell (mysophere) cultures, as the geometry of these cells simplifies the testing of theoretical predictions concerning the electromigration, diffusion, and aggregation of concanavalin A (con A) binding sites and AChRs (Orida and Poo, 1978, 1980, 1981; Poo et al., 1979; McLaughlin and Poo, 1981; Stollberg and Fraser, 1988).

We have shown previously that cultured Xenopus myospheres preserve at least some of the components required for the induction of receptor clustering (Stollberg and Fraser, 1988). AChRs accumulate at the cathode-facing cell pole in response to electric fields and continue to aggregate at that pole after the 


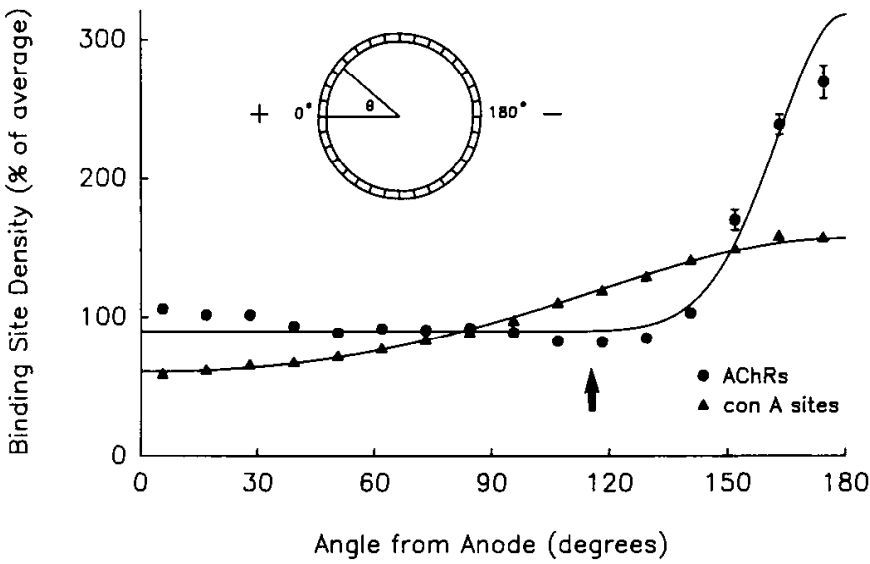

Figure 1. Distribution of AChRs and con A sites following an electric field of $8 \mathrm{~V} / \mathrm{cm}$ for $40 \mathrm{~min}$. Data are presented as means \pm standard errors of site density vs the angle $(\theta)$ from the anodal cell pole (see inset). In these control experiments, cells were not enzymatically pretreated. Circles, AChR distribution, $n=7$ cells. Arrow, Location of minimum AChR density. Curve, Theoretical fit to ideal distribution with $m / D=$ $577 \pm 36 V^{-1}, f_{\mathrm{m}}=0.10 \pm 0.005 ; \chi_{(13)}^{2}=141$, indicating that this curve does not adequately represent the data. Triangles, Con A site distribution, $n=11$ cells. Curve, Theoretical fit with $m / D=28 \pm 2 V^{-1}, f_{\mathrm{m}}$ $=0.68 \pm 0.05 ; \chi^{2}{ }_{(13)}=11$. This curve gives a good approximation of the observed distribution. Inset, Analysis of cell images. The perimeter was divided into 16 sectors according to the angle with respect to the electric field (the sectors are mirror-symmetric about the field axis). The average receptor density for the distribution shown is $100 \%$, although it may appear to be higher. This is because the sectors near the cell poles $\left(0,180^{\circ}\right)$ represent less of the sphere surface area than those near the equator. In order to calculate the total number of receptors on the cell surface, the intensity found at each sector is scaled by its surface area. Therefore, in calculating the average receptor density, the sectors near the poles are given less weight.

field has been terminated. ${ }^{1}$ The aggregation of receptors is specific; con A binding sites and lipids, which accumulate at the cathodal pole during exposure to a field, return to a uniform distribution after termination of the field (Poo et al., 1979; McLaughlin and Poo, 1981; Stollberg and Fraser, 1988). Receptor clustering in this system is consistent with a diffusion trap mechanism, is sensitive to trypsin digestion, and is insensitive to agents disrupting microtubules and microfilaments (Stollberg and Fraser, 1988). Together these results suggest that the aggregation of AChRs is mediated by adhesion or cohesion events on the extracellular face of the membrane. The simplicity and rapid response of the system (receptor clustering is triggered within $5 \mathrm{~min}$ ) render this a powerful experimental approach with which to probe the molecular events responsible for receptor clustering.

A simple and attractive model accounting for these observations is that the field-induced accumulation of AChRs at the cathodal pole triggers receptor aggregation. Under such a model, increased local density of receptors would play a causal role by shifting the equilibrium binding of receptors to one another, or to components in or adjacent to the cell membrane. This hypothesis is consistent with observations of AChR cluster formation in aneural cultures (Pumplin and Bloch, 1987), and with a widely held theory of patching and capping (Bourguignon and Bourguignon, 1984; see also Discussion). The rigorous exclusion

\footnotetext{
'We use "accumulation" or "concentration" to indicate a reversible increase in the density of a membrane component-due in our experiments to the application of electric fields. "Aggregation" or "clustering" is used to designate an irreversible association of membrane components.
}

of the hypothesis requires finding experimental conditions under which the aggregation of receptors can be dissociated from an antecedent increase in receptor density.

The search for such experimental conditions requires an understanding of the interactions between membrane components and electric fields. The driving force for the field-induced redistribution of membrane components ("clectromigration") is thought to be a combination of electrophoresis and electroosmosis (McLaughlin and Poo, 1981). Electroosmosis can be understood qualitatively by considering the negative charge bound to the extracellular membrane surface. As a consequence of this net negativity, positive ions in the saline are drawn to the membrane surface. These ions are highly mobile and in response to an electric field will induce a "solvent drag" in the direction of the cathodal pole. This drag, or electroosmotic force, acts on the extracellular aspect of membrane-bound molecules, pushing them in the cathodal direction. In this way molecules that carry a large amount of negative charge (relative to the average cell surface charge-the zeta potential) will migrate toward the anodal pole because the balance of the 2 forces favors electrophoresis. Other molecules with a lesser amount of negative charge will electromigrate toward the cathodal cell pole becausc clectroosmosis dominates. Evidence for the importance of the cell surface charge comes from experiments with neuraminidase, which removes negatively charged sialic acid residues from the cell surface. This reduces the magnitude of electroosmosis and should reverse the electromigration of some species from cathode-seeking to anode-seeking. The observed reversal of electromigration after such treatments supports the view that electroosmosis plays a significant role in electromigration (Orida and Poo, 1978; McLaughlin and Poo, 1981).

Manipulation of electromigration by neuraminidase, in combination with the spatial resolution of the imaging techniques used here, offers a possible means to separate receptor accumulation and receptor aggregation experimentally. The question addressed is whether the reversal of field-induced receptor migration is accompanied by reversed receptor aggregation. Our results indicate that AChRs become clustered at the cathodal pole (as in cells not treated with neuraminidase) despite reversal of receptor electromigration. Therefore, elevated receptor density does not trigger the aggregation of receptors.

\section{Materials and Methods}

Culture system. Myotomal cells were dissected from stage 18-20 Xenopus laevis embryos (Nieuwkoop and Faber, 1962) in Steinberg's solution, $\mathrm{pH} 7.8$, containing $1 \mathrm{mg} / \mathrm{ml}$ collagenase. The cells were dissociated in $\mathrm{Ca}^{2+} / \mathrm{Mg}^{2+}$-free Steinberg's solution, $\mathrm{pH} 7.8$, and maintained on sterile coverslips in drops of culture medium $(85 \%$ Steinberg's solution, $10 \%$ Leibovitz's L-15 medium, $5 \%$ fetal calf serum, $50 \mu \mathrm{g} / \mathrm{ml}$ gentamicin), $\mathrm{pH} 7.8$, for $1 \mathrm{~d}$ at $24^{\circ} \mathrm{C}$. For details of culture system, field application, and data analysis see Stollberg and Fraser (1988).

Experimental manipulations. Prior to the application of electric fields, cultures were either not treated in any way (controls), incubated with neuraminidase, or incubated with neuraminidase followed by trypsin. Incubations were carried out at room temperature in $50 \mu \mathrm{l}$ of Steinberg's solution at $\mathrm{pH} 6.6$ (neuraminidase) or $\mathrm{pH} 7.8$ (trypsin). The cultures were then returned to medium and assembled into electrophoresis chambers fashioned from a microscope slide, coverslip runners, and the coverslip culture (internal chamber dimensions $6.0 \mathrm{~cm} \times 1.0 \mathrm{~cm} \times 0.2$ $\mathrm{mm}$ deep). Electric potentials from an electrophoresis power supply were applied to the ends of the chambers via U-shaped 6-mm (I.D.) glass tubes filled with $2 \%$ agar/Steinberg's solution. Separate agar bridges were used to monitor the potential drop across the chambers, which offers a direct measure of the field strength. Following field application, and the indicated post-field relaxation periods (if any) at room temperature, the cells were chilled and labelcd for $10 \mathrm{~min}$ with $300 \mathrm{~nm}$ 

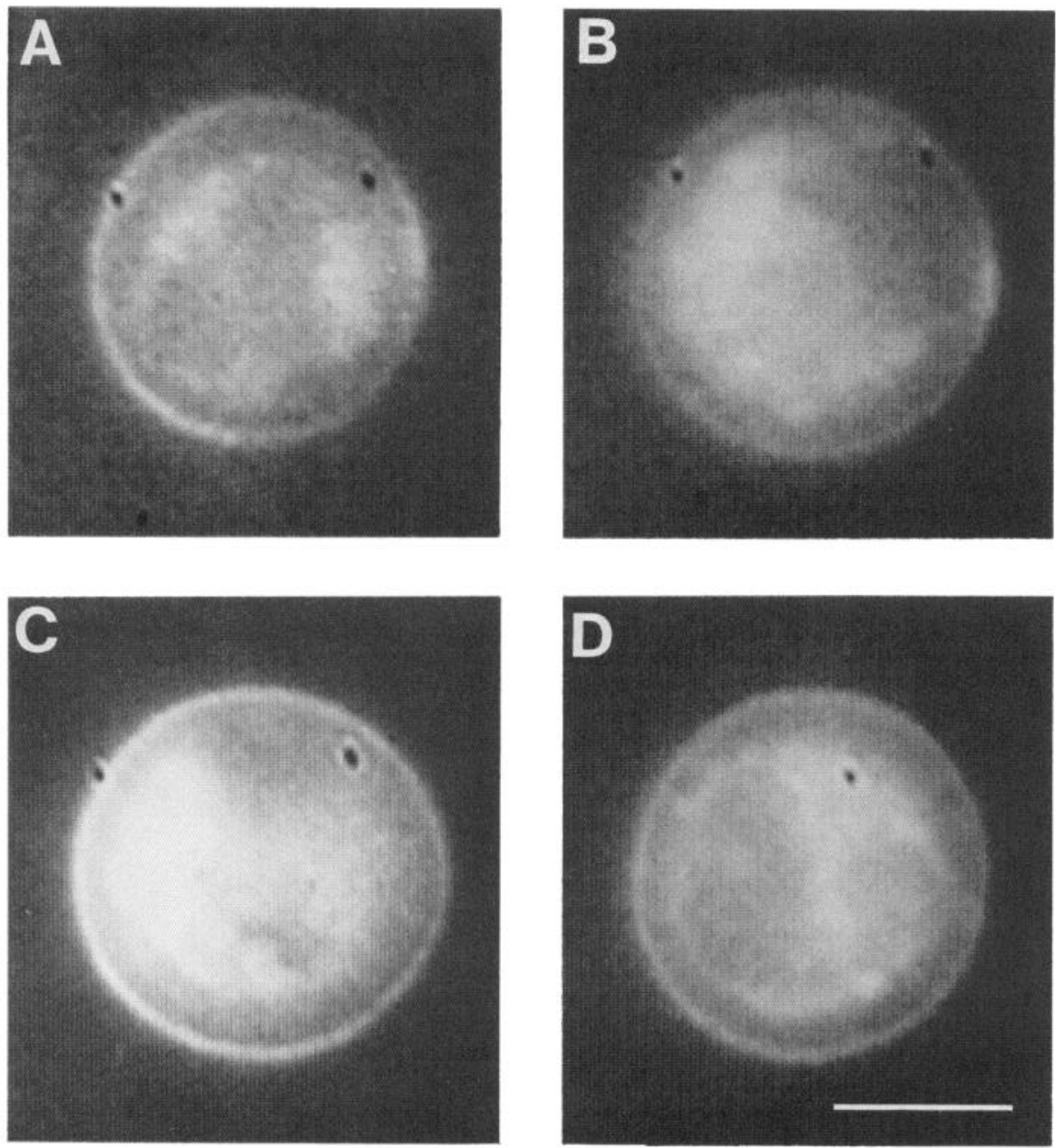

Figure 2. Representative fluorescence videomicrographs of cells labeled with TMR- $\alpha$-Bgt. These images are examples of the raw data and have not been corrected for fluorescence background or camera nonlinearities. The videomicrographs illustrate the visually modest changes in receptor distribution seen under the various conditions, while the more sensitive quantitative analyses make use of both the above-mentioned corrections and the averaging of many cell images (Figs. 1, 3-7). A, Receptor distribution on a cell treated with 0.2 $\mathrm{U} / \mathrm{ml}$ neuraminidase for $40 \mathrm{~min}$ and subjected to $8 \mathrm{~V} / \mathrm{cm}$ for $80 \mathrm{~min}$. Note the elevation of receptor density at both anode- and cathode-facing cell regions. $B$, As in $A$, but the cell was given 80 min of relaxation following field termination. Receptor aggregation has proceeded at the cathodal pole, while the density at the anodal pole has decreased. $C$, Receptor distribution on a cell pretreated with $0.2 \mathrm{U} / \mathrm{ml}$ neuraminidase for $40 \mathrm{~min}$, then with $0.1 \%$ trypsin for $20 \mathrm{~min}$, and then subjected to $8 \mathrm{~V} / \mathrm{cm}$ for $40 \mathrm{~min}$. Note the accumulation of receptors at the anode-facing pole and decrease at the cathodefacing pole. $D$, As in $C$, but the cell was given $80 \mathrm{~min}$ of relaxation following field termination. The receptor distribution is nearly uniform. The prominent dark spots in these images $(A-D)$ result from flaws in the camera tube; these along with other faulty pixels were rejected in the analysis. The bright regions in the cell interiors are due to autofluorescence (they appear even in unlabeled cells) and do not affect the quantitative image analysis. The field cathode is to the right in all cases. Calibration bar, $20 \mu \mathrm{m}$. rhodamine-labeled $\alpha$-Bgt (TMR- $\alpha$-Bgt), $25 \mu \mathrm{g} / \mathrm{ml}$ fluorescein-labeled concanavalin A (FLR-con A) in medium. The cultures were then rinsed and kept on ice until video images were acquired.

Data analysis. Cell images were gathered through a Zeiss Universal microscope, collected by a SIT video camera (RCA model No. TC1030), and stored on video cassettes (Sony U-matic video cassette recorder models VO-5600 and VO-5800). Videotape images were digitized using a Digisector DS-88 board (Microworks). Digital images were corrected on a pixel-by-pixel basis for spatial aberrations in the illumination, optics, and video tube. Pixels corresponding to the cell perimeter $( \pm 5 \%$ of the cell radius) were sorted into 16 sectors according to their angle with respect to the electric field (Fig. 1, inset). The intensities in these sectors were used to estimate the distribution of fluorescently labeled sites around the cell perimeter. Control experiments using known dye concentrations have confirmed the validity of the data-gathering and -analysis techniques (Stollberg and Fraser, 1988).

Theoretical considerations. Under "ideal" conditions (no interaction between the sites; spherical, nonconducting cells) the distribution of cell-surface sites at steady state in an electric field is a balance between electromigration and diffusion and has an analytical solution (Jaffe, 1977; Poo et al., 1979; McLaughlin and Poo, 1981; Ryan et al., 1988; Stollberg and Fraser, 1988). The form used here is that the site density as a function of $\theta\left(C_{\theta}\right)$ is

$$
C_{\theta}=f_{\mathrm{m}} \cdot \alpha \cdot \exp (-\beta \cdot \cos \theta)+\left(1-f_{\mathrm{m}}\right) \cdot C_{\mathrm{i}}
$$

where $\alpha=\beta \cdot C_{\mathrm{i}} / \sinh (\beta), \beta=1.5 \cdot \mathrm{E} \cdot r \cdot m / D, \theta$ is the angular position relative to the anodal cell pole, $r$ is the cell radius, $C_{i}$ the initial molecular density, $E$ the field strength, $f_{\mathrm{m}}$ the fraction of mobile sites, $m$ the electromigrational mobility constant, and $D$ the diffusion constant. The 2 parameters $m / D$ and $f_{\mathrm{m}}$ were allowed to vary in fitting this description to data (all other parameters being measured quantities).

\section{Results}

The experimental design utilizes enzymatic treatments and electric fields to study the migration and clustering of AChRs on cultured Xenopus myospheres. Following these manipulations, the distribution of fluorescently labeled membrane components was quantified from digitized video images (see Fig. 1, inset, and Material and Methods). As a basis of comparison to enzymatically treated cells, untreated cultures were subjected to electric fields and analyzed for the distribution of con A sites and AChRs. The results (Fig. 1) represent the 2 kinds of distributions documented previously (Stollberg and Fraser, 1988). The con A site distribution is as expected for "ideal" (noninteracting) sites in that the data are well described by a theoretical analysis which assumes that electromigration and diffusion dominate the process (see Materials and Methods, Theoretical considerations). Furthermore, the same parameters fit the steadystate con A site distributions over a 4-fold range in field strengths, and the distributions decay back to uniformity after termination of the field (Stollberg and Fraser, 1988). Thus, the con A sites 


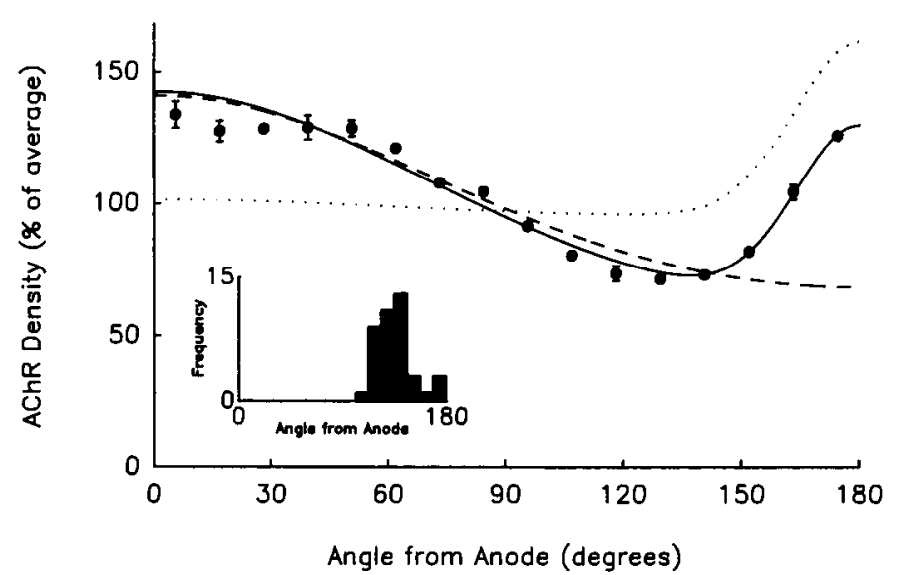

Figure 3. Distribution of AChRs following neuraminidase treatment and exposure to electric fields. Cells were treated with $0.2 \mathrm{U} / \mathrm{ml} \mathrm{neur-}$ aminidase for $40 \mathrm{~min}$ and subjected to a field of $8 \mathrm{~V} / \mathrm{cm}$ for $80 \mathrm{~min}$. The distribution is bimodal, indicating receptor accumulations at both anode- and cathode-facing poles. Solid curve, Theoretical fit for the sum of 2 ideally behaving populations: first population, $m / D=632 \pm 54$ $V^{-1}, f_{\mathrm{m}}=0.027 \pm 0.002$; second population, $m / D=-21 \pm 1 V^{-1}, f_{\mathrm{m}}$ fixed at 0.97 (else the algorithm forced the value over 1.0 ); $\chi_{(12)}^{2}=64$. Dashed curve, Theoretical result fit by eye to left half of the data, yielding $m / D=-19 V^{-1}\left(f_{\mathrm{m}}=0.77\right.$, from data of Fig. 6). Dotted curve, Estimated distribution of AChRs resulting from aggregation alone-this curve is the difference between the solid and dashed curves (see Results). Inset, Frequency histogram showing the positions of minimal receptor density among the cells. Data are means \pm standard errors from 4 experiments, each consisting of 9-11 scanned cells.

behave in accordance with the theoretical predictions under these conditions.

In contrast, the ficld-induced distribution of AChRs is markedly nonideal (Fig. 1). It deviates substantially from the bestfitting theoretical curve, with a characteristic minimum at about $120^{\circ}$ (Fig. 1, arrow). Moreover, the receptors continue to aggregate at the cathodal pole after termination of the field (Stollberg and Fraser, 1988). This finding, and the proximity of minimum receptor density to the forming aggregate, are consistent with the action of a specific diffusion trap for AChRs at the cathodal pole of the cell. AChRs clearly behave nonideally; their

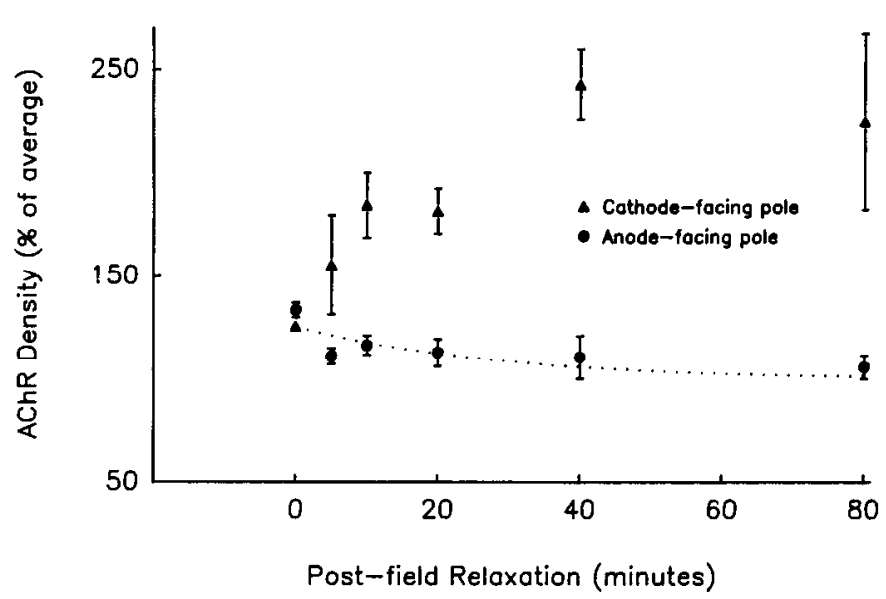

Figure 4. Density of AChRs at the 2 cell poles as a function of time, following termination of the electric field. Cells were incubated with 0.2 $\mathrm{U} / \mathrm{ml}$ neuraminidase for $40 \mathrm{~min}$, subjected to $8 \mathrm{~V} / \mathrm{cm}$ for $40 \mathrm{~min}$, and given the indicated times for postfield relaxation. Circles, Receptor density at the anode-facing cell pole. Dotted curve, Exponential with time constant $27.8 \mathrm{~min}$, corresponding to $D=1.2 \times 10^{-9} \mathrm{~cm}^{2} / \mathrm{sec}$ (see Fig. 6 , legend $; \chi_{(3)}^{2}=12$. The rate of decrease in receptor density at the anode is consistent with the given diffusion constant. Triangles, Receptor density at the cathode-facing pole. Data are means \pm standard errors from 3-6 experiments, each consisting of 6-11 analyzed cells.

distribution reflects a combination of electromigration, diffusion, and (predominantly) receptor aggregation (Stollberg and Fraser, 1988). The simplest hypothesis to explain receptor aggregation is that it is triggered by the electromigrational increase in receptor density at the cathode-facing pole.

To test the hypothesis that field-induced accumulation of AChRs causcs the receptor trap to form, cells were subjected to neuraminidase treatments reported previously to reverse the electromigration of membrane components (Orida and Poo, 1978; McLaughlin and Poo, 1981). An example of a cell incubated with neuraminidase, placed in an electric field, and labeled with TMR- $\alpha$-Bgt is shown in Figure $2 A$. Quantitative analysis of many such images shows clearly that the receptor distribution is bimodal, with increased density at both the anodal and cathodal cell poles (Fig. 3). The frequency histogram of minimum

Table 1. Asymmetry indices following various neuraminidase treatments

\begin{tabular}{|c|c|c|c|c|c|}
\hline \multirow[b]{2}{*}[\text{Neur}]{$^{a}$} & \multirow[b]{2}{*}{$\mathrm{N}^{\prime \prime}$} & \multicolumn{2}{|l|}{ AChRs } & \multicolumn{2}{|l|}{ con A sites } \\
\hline & & $\overline{A_{\text {sector }}}$ & $A_{\text {rectangle }}$ & $\overline{A_{\text {sccior }}}$ & $A_{\text {rectanglc }}$ \\
\hline 0 & c & $0.43 \pm 0.01$ & $0.24 \pm 0.02$ & $0.46 \pm 0.02$ & $0.41 \pm 0.02$ \\
\hline 0.05 & 8 & $-0.06 \pm 0.05$ & $-0.12 \pm 0.04$ & $0.13 \pm 0.04$ & $0.08 \pm 0.05$ \\
\hline 0.2 & 9 & $-0.13 \pm 0.06$ & $-0.20 \pm 0.03$ & $0.10 \pm 0.02$ & $0.06 \pm 0.02$ \\
\hline 1.0 & 17 & $0.00 \pm 0.00$ & $0.01 \pm 0.01$ & $0.13 \pm 0.01$ & $0.09 \pm 0.02$ \\
\hline 2.0 & 14 & $-0.10 \pm 0.02$ & $-0.10 \pm 0.02$ & $0.07 \pm 0.03$ & $0.06 \pm 0.03$ \\
\hline
\end{tabular}

Cells were incubated with the given concentrations of neuraminidase for $80 \mathrm{~min}$ and then subjected to fields of $8 \mathrm{~V} / \mathrm{cm}$ for $40 \mathrm{~min}$. The resulting distributions are summarized by the asymmetry index $(A) ; A=\left(C_{\mathrm{C}}-C_{\mathrm{A}}\right) /\left(C_{\mathrm{C}}+C_{\mathrm{A}}\right)$, where $C_{\mathrm{A}}$ and $C_{\mathrm{C}}$ are the densities at the anodal and cathodal poles, respectively. $A_{\text {sector }}$ is the asymmetry index calculated using the densities at sectors 1 and 16 as estimates for $C_{\mathrm{A}}$ and $C_{\mathrm{C}}$. $A_{\text {recange }}$ is calculated using rectangles $(\mathrm{ca} .3 .5 \times 18 \mu \mathrm{m})$ as estimates for $C_{\mathrm{A}}$ and $C_{\mathrm{C}}$ and is presented to facilitate comparison with previously reported results. The 2 kinds of asymmetry indices give approximately the same values in the experiments summarized here. For both $\mathrm{AChRs}$ and con A sites, the indices are markedly reduced by neuraminidase treatment. The AChR distributions reverse in polarity by this measure, while con A site distributions approach neutrality. Values are means \pm standard errors and have been rounded to 2 decimal places.

"Neuraminidase concentration in units $/ \mathrm{ml}$.

"Number of cells analyzed.

c Same experiments as Figure 2; the cultures were labeled with $300 \mathrm{nM}$ TMR- $\alpha$-Bgt ( $n=7$ cells) or $25 \mu \mathrm{g} / \mathrm{ml}$ TMR-con A $(n=11$ cells $)$ 


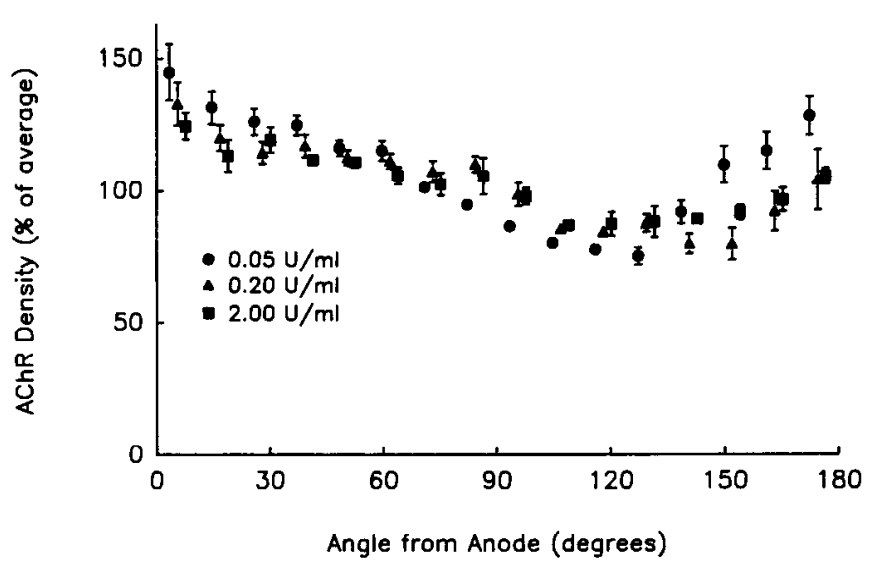

Figure 5. Field-induced AChR distributions following progressively more extensive neuraminidase digestions. Cells were incubated with the indicated concentrations of neuraminidase for $80 \mathrm{~min}$ and then subjected to a field of $8 \mathrm{~V} / \mathrm{cm}$ for $40 \mathrm{~min}$. Although there may be some differences in the distributions, the significant features remain unaltered. All 3 distributions show the characteristic bimodality consistent with receptor electromigration toward the anode and receptor aggregation at the cathode. Data are means \pm standard errors for 6-9 cells per condition.

density location shows that the bimodality of the AChR distribution is a feature common to most individual cells, rather than an artifact resulting from the averaging of 2 cell populations with density minima at opposite poles (Fig. 3, inset).

The bimodality of this AChR distribution implies 2 opposed processes. The density profile near the anodal pole is reminiscent of an ideal distribution, while that near the cathodal pole suggests the operation of a diffusion trap (compare to Fig. 1). If this interpretation is correct, it should be possible to decompose the overall distribution into these 2 components. An estimate of the contribution made by ideal behavior is shown in Figure 3 , dashed curve. The difference between this estimate and the original distribution is shown by the dotted curve, which is similar to the AChR distribution (dominated by receptor aggregation) shown in Figure 1. This analysis strengthens the interpretation that the data of Figure 3 represent the sum of 2 opposed processes: ideal electromigration (toward the anodal cell pole) and field-induced aggregation of receptors (at the cathodal pole).

To further test this interpretation, the AChR distribution on neuraminidase-treated cells was monitored following a postfield relaxation period (Fig. $2 B$ ). Results averaged from several such experiments show that the AChR density continued to increase only at the cathodal pole, as in cells not treated with neuraminidase (Fig. 4). Thus, the AChR aggregation event is confined to the cathode-facing pole, consistent with the interpretation given the data of Figure 3 . The average receptor density at the anode-facing pole was as large as that at the cathodefacing pole immediately following the field (Figs. 3, 4). However, the anodal density decayed back to control levels after termination of the field (Fig. 4). The simplest interpretation of this result is that elevated AChR density in and of itself is not sufficient to induce receptor aggregation (see also Discussion).

Another possible explanation for the data of Figures 3 and 4 would be that the conditions of neuraminidase treatment resulted in a class of digested receptors that is incompetent with respect to aggregation and an undigested class that is still able to aggregate. This could be attributed to the action of neur-

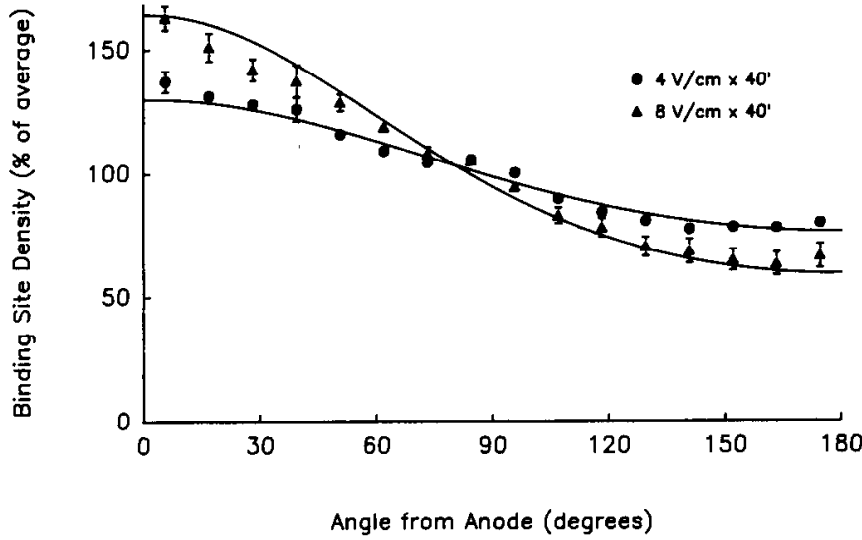

Figure 6. Steady-state distributions of $\mathrm{AChRs}$ following treatment with neuraminidase and trypsin. Cells were incubated with $0.2 \mathrm{U} / \mathrm{ml}$ neuraminidase for $40 \mathrm{~min}, 0.1 \%$ trypsin for $20 \mathrm{~min}$, and then exposed to electric fields for $40 \mathrm{~min}$. Circles, $4 \mathrm{~V} / \mathrm{cm}$. Triangles, $8 \mathrm{~V} / \mathrm{cm}$. Curves, Theoretical fit to ideal distributions; $m / D=-34 \pm 4 V^{-1}, f_{\mathrm{m}}=0.77 \pm$ $0.08, \chi^{2}{ }_{(29)}=117$. The same parameters fit the 2 distributions reasonably well, though the possibility of nonideal behavior at the higher field strength cannot be eliminated (see Discussion). Data are means \pm standard errors from 3-4 experiments, each consisting of 5-12 analyzed cells.

aminidase itself or to a contaminant activity in the enzyme preparation. According to this "partial digestion" scenario, the elevated receptor density at the anodal pole (Fig. 3) consists of the digested receptors, which consequently fail to aggregate after

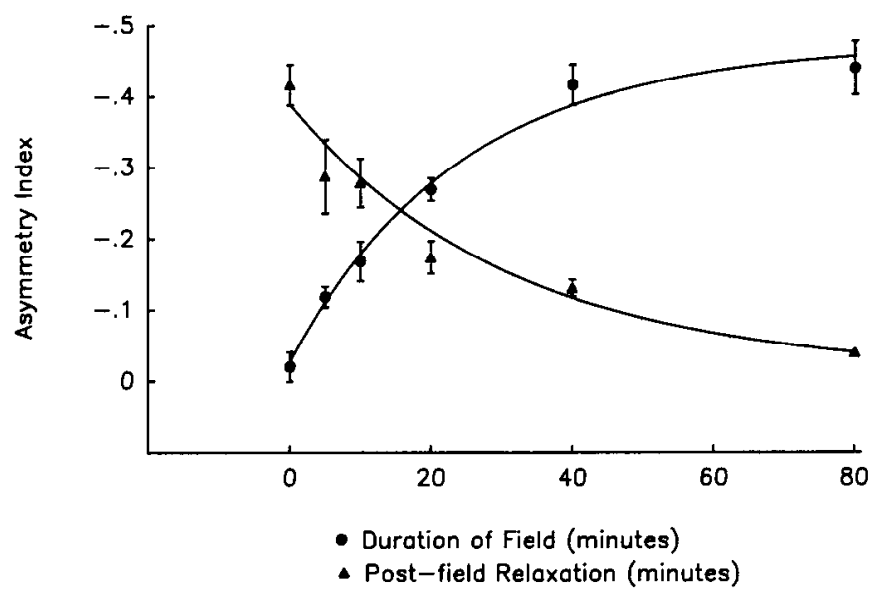

Figure 7. Change in AChR asymmetry as a function of time. The asymmetry index $(A)$ is determined by the densities at the anodal $\left(C_{\mathrm{A}}\right)$ and cathodal $\left(C_{\mathrm{C}}\right)$ cell poles and is defined as $A=\left(C_{\mathrm{C}}-C_{\mathrm{A}}\right) /\left(C_{\mathrm{C}}+C_{\mathrm{A}}\right)$. Densities at sectors 1 and 16 were used to estimate $C_{\mathrm{A}}$ and $C_{\mathrm{C}}$. Cells were incubated with $0.2 \mathrm{U} / \mathrm{ml}$ neuraminidase for $40 \mathrm{~min}, 0.1 \%$ trypsin for $20 \mathrm{~min}$, and then subjected to fields of $8 \mathrm{~V} / \mathrm{cm}$. Circles, Asymmetry development with time in the field. Cells were subjected to the indicated field duration and analyzed for sector asymmetry. Curve, Best-fit exponential to the data; time constant $=24 \pm 5 \mathrm{~min}, \chi_{(3)}^{2}=2$. The receptor diffusion constant $(D) \approx r^{2}\left(1-0.1 \cdot \beta^{2}\right) / 2 \cdot \tau$ (Poo, 1982). Given $m / D$ $\approx-34 \mathrm{~V}^{-1}$ (Fig. 6, legend), $r=20 \mu \mathrm{m}$, and $E=8 \mathrm{~V} / \mathrm{cm}, \beta$ is approximately -0.8 . Together with the observed $\tau$, this gives an estimate of $D \approx 1.3( \pm 0.2) \times 10^{-9} \mathrm{~cm}^{2} / \mathrm{sec}$. Triangles, Asymmetry decay with time postfield. Cells were subjected to the field and then given the indicated times for postfield relaxation. Curve, Best-fit exponential to the data; time constant $=31 \pm 5 \mathrm{~min}, \chi^{2}{ }_{(3)}=6$. The decay of asymmetry is approximated by an exponential such that $D=r^{2} / 2 \cdot \tau$ (Huang, 1973). This leads to an estimate for $D$ of $\approx 1.1( \pm 0.2) \times 10^{-9} \mathrm{~cm}^{2} / \mathrm{sec}$. Data are means \pm standard errors from 3-4 experiments, each consisting of $7-11$ analyzed cells. 
field termination (Fig. 4). If the standard incubation conditions result in a partial digestion phenomenon as outlined above, then more extensive digestion should eliminate all aggregation activity, resulting in a distribution that is ideal and maximal at the anodal cell pole. As shown in Figure 5, such distributions were not seen. Instead, incubation conditions that should yield 20 times the digestion of the standard conditions result in bimodal distributions, showing that AChRs still aggregate at the cathodal cell pole. The marginal changes in the field-induced distribution of AChRs with the different neuraminidase concentrations (Fig. 5) suggest that the standard incubation conditions are sufficient to remove nearly all of the susceptible negative charges from the cell surface. In support of this, the electromigration of con A binding sites, which is altered by the standard incubation conditions, is not altered further by more cxtcnsive ncuraminidase digestion (Table 1). These results argue strongly that partial digestion of receptors by the neuraminidase preparation is not responsible for the failure of AChRs to aggregate at the anodal pole.

AChR aggregation, seen as a consequence of exposure to electric fields, can be blocked by mild digestion with trypsin (Stollberg and Fraser, 1988). If the data of Figure 3 represent the opposed actions of electromigration and receptor aggregation, blockage of receptor aggregation by trypsin treatment should result in an ideal receptor distribution with a maximum at the anodal cell pole. This prediction is borne out by experiments in which cells were treated with neuraminidase and trypsin before field application (Figs. 2C,6). These distributions are well fit by the theoretical predictions for ideally behaving sites (Fig. 6 , legend). The distributions support the conclusion that the direction of $\mathrm{AChR}$ electromigration has indeed been reversed by neuraminidase treatment.

Because AChRs behave ideally after treatment with neuraminidase and trypsin, the development and decay of asymmetry can be used to estimate the receptor diffusion constant (D). Rates of development and decay of AChR asymmetries are shown in Figure 7. These time courses are well fit by exponentials indicating that $D \approx 1.3 \times 10^{-9} \mathrm{~cm}^{2} / \mathrm{sec}$ (asymmetry development), and $D \approx 1.1 \times 10^{-9} \mathrm{~cm}^{2} / \mathrm{sec}$ (asymmetry decay). The agreement between these 2 estimates is noteworthy and is consistent with the characterization of AChR behavior as ideal following incubation with neuraminidase and trypsin.

\section{Discussion}

This study focuses on the initial AChR clustering events in a model system-cultured Xenopus myospheres exposed to electric fields. Receptor aggregation is suggested by the nonideal behavior of AChRs in response to such fields (Fig. 1). Other experiments have shown that AChRs continue to aggregate at the cathode-facing pole after the field is terminated (Stollberg and Fraser, 1988). These results are consistent with a slight electromigrational accumulation of receptors at the cathodefacing pole, superimposed on a much larger accumulation due to specific receptor trapping. The trap is selective for receptors and is triggered in the absence of neurons, surface contacts, or exogenous tissue-derived factors, rendering this a useful model system in which to study mechanisms of receptor aggregation on the molecular level.

In the present work we have tested a simple and attractive hypothesis to explain the field-induced triggering of AChR aggregation (there are only 2 other possibilities-see below). The hypothesis holds that receptor aggregation is triggered by locally increased receptor density, due in these experiments to electromigration (Poo, 1982; Stollberg and Fraser, 1988; for theoretical framework see Gershon, 1978). This view is consistent with the observation that transient, local increases in receptor density precede the sorting out of receptors into a "lattice" arrangement during cluster formation at myotube-substrate contacts (Pumplin and Bloch, 1987) and has been incorporated into a scheme accounting for the contact-mediated induction of receptor clusters (Bloch and Pumplin, 1988). The hypothesis is also consistent with the much-studied phenomenon of capping, which is caused by the externally induced rearrangement (patching) of cell surface molecules by multivalent ligands (Taylor et al., 1971; de Petris and Raff, 1973; for review see Bourguignon and Bourguignon, 1984). Other experimental approaches to receptor clustering have suggested a requirement for complex interactions with other molecules, which might appear to rule out the hypothesis under test here. In particular, there is considerable evidence for the involvement of cytoskeletal anchoring of $A C h R$ clusters some hours after formation (Bloch and Hall, 1983; Bloch, 1986; Podleski and Salpeter, 1988). However, there is no compelling evidence for the causal role of these connections in initial clustering events (see Kuromi et al., 1985). Moreover, it has been shown previously that cytoskeleton-disrupting drugs do not reduce the field-induced aggregation of receptors in Xenopus myosphere cultures (Orida and Poo, 1978, 1980; Stollberg and Fraser, 1988). Finally, we know that cytoskeletal anchoring cannot be the initial clustering event at nerve-muscle contacts in vivo. This is because the signal for receptor clustering arises from interactions between the muscle cell and motor neuron and must therefore be transduced by some combination of binding by tissue-derived factors (e.g., Godfrey et al., 1984; Usdin and Fischbach, 1986), contact-mediated events (Peng et al., 1981; Bloch and Pumplin, 1988), or endogenous electric fields (Fraser and Poo, 1982).

Neuraminidase, which removes sialic acid residues and alters the surface charge of the cell, has been reported to reverse the field-induced migration of AChRs and con A sites (Orida and Poo, 1978; McLaughlin and Poo, 1981). This suggests a means to test the experimental hypothesis directly, by determining whether such conditions reverse receptor electromigration, receptor aggregation, or both. The experiments described here indicate that neuraminidase-treated cultures do show reversed electromigration of AChRs, but that receptor aggregation continues to take place at the cathodal pole, as in control (enzymatically untreated) cultures.

The distribution of AChRs, following neuraminidase treatment and exposure to electric fields, is bimodal; the density is elevated at both cell poles (Fig. 3). This is a property of most individual cells, rather than a consequence of 2 or more cell populations (Fig. 3, inset). The observation of a bimodal distribution is a novel finding and depends on the spatially resolved quantitation of receptor density employed in these experiments. Earlier experiments, in which distributions were characterized by an asymmetry index based solely on density at the cathodal and anodal ccll polcs, cmphasized the reversal of field-induced asymmetry following neuraminidase digestion (Orida and Poo, 1978; McLaughlin anad Poo, 1981). We find that the receptor asymmetry reverses, as shown in Table 1 , in accord with the previous work. The con A site asymmetry decreased to near zero but did not actually reverse in response to neuraminidase digestion (Table 1). A difference as small as 10-20\% in the charge susceptible to neuraminidase cleavage could account for this 
minor discrepancy. Given the possible differences in cultures and reagents, this small difference between our results and those reported previously is probably not important.

After termination of the field, neuraminidase-treated cultures display continued receptor aggregation at the cathodal cell pole, but the elevated receptor density at the anodal pole decreases (Fig. 4). This suggests that elevated receptor density is not sufficient to trigger receptor aggregation. It might be thought that partial digestion could account for this result if more digested receptors are not competent with respect to aggregation. The argument would hold that native $\mathrm{AChRs}$ electromigrate to the cathodal cell pole and are triggered to aggregate by their elevated density there; in contrast, digested receptors electromigrate to the anodal pole and cannot aggregate. It is difficult to reconcile this scenario with the charge-removing effects of neuraminidase; receptors that have been more extensively cleaved of sialic acid residues will be less negatively charged and will therefore migrate preferentially toward the cathodal cell pole compared to native AChRs. Moreover, the partial digestion scenario is inconsistent with the finding that $20 \times$ greater digestion with neuraminidase still yields a bimodal field-induced distribution of AChRs (Fig. 5, Table 1). Thus, the results cannot be explained by invoking partial digestion of receptors, whether by the neuraminidase itself or by a contaminant in the preparation.

Treatment of cells with trypsin has been shown to block receptor aggregation, and to have little if any effect on the electromigration of receptors (Stollberg and Fraser, 1988). If the bimodal receptor distributions in neuraminidase-treated cells reflect the opposition of electromigration and receptor aggregation, cells incubated with neuraminidase and trypsin should show ideal AChR distributions with the maximal density at the anodal cell pole. In agreement with this expectation, the distributions seen under these conditions are consistent with the predictions of ideal electromigration (Fig. 6). The distribution seen at $8 \mathrm{~V} / \mathrm{cm}$ is slightly less asymmetric than would be predicted on the basis of the $4 \mathrm{~V} / \mathrm{cm}$ data, which may reflect the presence of nonideal interactions between receptors at the higher field strength (Ryan et al., 1988; Stollberg and Fraser, 1988). These results support the view that neuraminidase treatment reverses electromigration of receptors, so that receptor density is electromigrationally lowered at the cathodal cell pole. Thus, the formation of receptor aggregates at the cathodal pole after neuraminidase digestion indicates that elevated receptor density is not necessary to trigger receptor aggregation.

Because treatment of the cells with trypsin creates ideally behaving receptors, the rates at which asymmetries develop during field administration, and decay after field termination, can be used to estimate the receptor diffusion constant. In principle, such kinetic studies could be performed with trypsin treatment alone; however, the electromigration of AChRs under these conditions is too small to permit accurate measurements (Stollberg and Fraser, 1988). This limitation is circumvented by combined neuraminidase and trypsin treatments, which result in an easily measurable electromigration of AChRs. The results of such cxperiments provide an estimated diffusion constant of about $1.2 \times 10^{-9} \mathrm{~cm}^{2} / \mathrm{sec}$ (Fig. 7, legend). Although the AChRs are 2 enzymatic steps removed from native receptors, the alteration of receptor diffusion is probably minimal. Trypsin digestion leaves the function and $\alpha$-Bgt binding of the receptor intact (Stollberg and Fraser, 1988), and digestion with other proteases reveals that AChRs are resistant to changes in function, size, shape, and sedimentation under nondenaturing con- ditions (Lindstrom et al., 1980). Neuraminidase digestion had no effect on $\alpha$-Bgt binding (data not shown) and should not materially affect the receptor mass. Therefore, the AChR diffusion constant measured in these experiments is probably close to that for native receptor.

Estimates of the AChR diffusion rate are critical to an understanding of the molecular mechanisms responsible for receptor clustering. In particular, the diffusion trap hypothesis assumes that receptor diffusion is rapid enough to permit significant numbers of AChRs to be trapped within their average lifetime (Fraser and Poo, 1982; Poo, 1982; Kuromi et al., 1985; Stollberg and Fraser, 1988). The diffusion constant reported here $\left(D \approx 1.2 \times 10^{-9} \mathrm{~cm}^{2} / \mathrm{sec}\right)$ is easily large enough to account for the receptor aggregation seen in Xenopus myosphere cultures (Stollberg and Fraser, 1988). The value is similar to that found for AChRs on cultured Xenopus muscle cells $\left(2.6 \times 10^{-9} \mathrm{~cm}^{2 /}\right.$ sec; Poo, 1982), and for extrajunctional AChRs in Xenopus

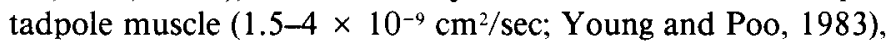
as measured by the diffusion of functional AChRs into a region of toxin-inactivated receptors. It is also similar to estimated diffusion constants for plasma membrane antigens on cultured embryonic muscle cells as measured by the diffusion of a locally labeled population (1-3 $\times 10^{-9} \mathrm{~cm}^{2} / \mathrm{sec}$; Edidin and Fambrough, 1973), and to that of con A sites on cultured Xenopus muscle cells $\left(2.3 \times 10^{-9} \mathrm{~cm}^{2} / \mathrm{sec}\right.$; Stollberg and Fraser, 1988$)$.

However, our estimate is considerably higher than the receptor diffusion constant determined by photobleaching recovery methods for Xenopus muscle cultures $\left(2.5 \times 10^{-10} \mathrm{~cm}^{2} / \mathrm{sec}\right.$; Kuromi et al., 1985) and cultured rat myotubes $\left(5 \times 10^{-11} \mathrm{~cm}^{2} /\right.$ sec; Axelrod et al., 1976). It has been suggested that diffusion estimates based on photobleaching may be smaller than those based on the decay of a gradient because intermolecular interactions lead to different rates of "self" vs "mutual" diffusion (Scalettar et al., 1988). Our diffusion measurements, performed in the presence of gradients, would presumably reflect a measure of mutual diffusion. However, the estimates that are in agreement with our own (Edidin and Fambrough, 1973; Poo, 1982; Young and Poo, 1983) were obtained in the absence of gradients and are therefore measures of self-diffusion. Thus, it appears that the distinction between mutual and self-diffusion cannot account for the discrepancy between our values and those obtained by photobleaching methods.

\section{The triggering of receptor aggregation}

The simple hypothesis that a homogeneous AChR population is triggered to aggregate by increased local density is excluded by the results discussed above. The data of Figure 4 show that AChR density at the anodal pole is elevated immediately after field termination, but decreases during the postfield period. Thus, elevated receptor density is not sufficient to induce aggregation. Furthermore, receptor aggregation is initiated at the cathodefacing pole despite the electromigrational lowering of $\mathrm{AChR}$ density (evident when receptor aggregation is blocked; rig. 6). Therefore, local elevation of receptor density is not necessary to trigger receptor aggregation.

There remain only 2 classes of mechanisms for the triggering of receptor aggregation that are consistent with our results. First, receptor aggregation may be triggered by the electromigrational accumulation of some molecule(s) as yet not probed for in this model system. Work underway in several laboratories is directed toward characterization of tissue-derived factors that induce AChR aggregates in vitro and may play a role in AChR clustering 
in vivo (e.g., Godfrey et al., 1984; Usdin and Fischbach, 1986; for review see Schuetze and Role, 1987). The in vitro action of such factors clearly requires interaction with cell-surface components; an attractive possibility is that the same components are responsible for the accumulation documented here. Another possibility is that the "other molecules" are in fact a distinct receptor subpopulation. This seems unlikcly, as it would require significant differences in the properties of receptor subtypes (i.e., aggregation potential, extracellular charge, susceptibility to neuraminidase digestion), but the possibility cannot be rigorously excluded.

A second class of mechanism for the triggering of receptor aggregation is suggested by the depolarization of the cell membrane that is associated with the application of electric fields. At $8 \mathrm{~V} / \mathrm{cm}$, a myosphere with a radius of $20 \mu \mathrm{m}$ will be depolarized by about $16 \mathrm{mV}$ at the cathode-facing pole. Thus, voltage-sensitive mechanisms must be considered a possible trigger for receptor aggregation. The data could be explained, under such a scenario, if the adhesion or cohesion events responsible for receptor aggregation were controlled in a voltage-dependent manner.

We attempted to distinguish between the 2 hypotheses (molecular accumulation vs voltage-dependent action) by searching for neuraminidase treatment conditions under which receptor aggregation is reversed (i.e., aggregation occurs at the anodal pole). As such treatments affect the electromigrational driving force, but not local membrane potentials, reversed receptor aggregation would rule out the direct involvement of voltagesensitive mechanisms. We have examined AChR distributions in cells incubated at up to $2 \mathrm{U} / \mathrm{ml}$ neuraminidase without observing such a reversal of aggregation (Table 1). It may be that the extracellular charge on the molecules that trigger receptor aggregation is such that even extensive treatment cannot cause reversal of their electromigration; hence this negative result does not distinguish between the 2 hypotheses. Accordingly, experiments based on other distinct predictions made by the 2 hypotheses must be performed. The current experimental system is ideally suited to further examination of the molecular mechanisms of rcceptor aggregation by virtue of the simplicity and rapid response of the system, as well as the quantitative nature of the analysis.

\section{Summary of conclusions}

Receptor aggregation can be initiated independently of cell-substrate contacts and soluble factors in a simple model system. This indicates that the system preserves some endogenous, surface-associated components that are sufficient to induce local, specific, receptor clustering. Neuraminidase treatment reverses the electromigration of $\mathrm{AChRs}$, which is consequently directed toward the anodal pole, but leaves unchanged the receptor aggregation event at the cathode-facing pole. After termination of the electric field, receptor density in these cultures decreases at the anodal pole, but continues to increase at the cathodal pole. Therefore, the local accumulation of AChRs is neither necessary nor sufficient to trigger the receptor aggregation event. With the elimination of this hypothesis, only 2 possible causes of receptor aggregation in this model system remain: (1) field-induced accumulation of some other molecule(s), or (2) a voltage-sensitive mechanism.

\section{References}

Anderson, M. J., and M. W. Cohen (1977) Nerve-induced and spontaneous redistribution of acetylcholine receptors on cultured muscle cells. J. Physiol. Lond. 268: 757-73.

Axelrod, D., P. Ravdin, D. E. Koppel, J. Schlessinger, W. W. Webb, E. L. Elson, and T. R. Podleski (1976) Lateral motion of fluorescently labeled acetylcholine receptors in membranes of developing muscle fibers. Proc. Natl. Acad. Sci. USA 73: 4594-4598.

Blackshaw, S., and A. Warner (1976) Onset of acetylcholine sensitivity and endplate activity in developing myotome muscles of Xenopus. Nature 262: 217-218.

Bloch, R. J. (1986) Actin at receptor-rich domains of isolated acetylcholine receptor clusters. J. Cell Biol. 102: 1447-1458.

Bloch, R. J., and Z. W. Hall (1983) Cytoskeletal components of the vertebrate neuromuscular junction: Vinculin, $\alpha$-actinin, and filamin. J. Cell Biol. 97: 217-223.

Bloch, R. J., and D. W. Pumplin (1988) Molecular events in synaptogenesis: Nerve-muscle adhesion and postsynaptic differentiation Am. J. Physiol. 254: c345-c364.

Bourguignon, L. Y. W., and G. J. Bourguignon (1984) Capping and the cytoskeleton. Int. Rev. Cytol. 87: 195-224.

Creazzo, T. L., and G. S. Sohal (1983) Neural control of embryonic acetylcholine receptor and skeletal muscle. Cell Tissue Res. 228: 112.

Dennis, M. J. (1981) Development of the neuromuscular junction: Inductive interactions between cells. Annu. Rev. Neurosci. 4: 43-68. dc Pctris, S., and M. C. Raff (1973) Normal distribution, patching and capping of lymphocyte surface immunoglobulin studied by electron microscopy. Nature (Lond.) New Biol. 241: 257-259.

Edidin, M., and D. Fambrough (1973) Fluidity of the surface of cultured muscle fibers. J. Cell Biol. 57: 27-37.

Edwards, C., and H. L. Frisch (1976) A model for the localization of acetylcholine receptors at the muscle endplate. J. Neurobiol. $7: 377-$ 381.

Frank, E., and G. D. Fischbach (1979) Early events in neuromuscular junction formation in vitro. Induction of acetylcholine receptor clusters in the postsynaptic membrane and morphology of newly formed nerve-muscle synapses. J. Cell Biol. 83: 143-58.

Fraser, S. E., and M.-M. Poo (1982) Development, maintenance, and modulation of patterned membrane topography: Models based on the acctylcholine receptor. Cur. Top. Dev. Biol. 17: 77-100.

Gershon, N. D. (1978) Model for capping of membrane receptors based on boundary surface effects. Proc. Natl. Acad. Sci. USA 75: $1357-1360$.

Godfrey, E. W., R. M. Nitkin, B. G. Wallace, L. L. Rubin, and U. J. McMahan (1984) Components of Torpedo electric organ and muscle that cause aggregation of acetylcholine receptors on cultured muscle cells. J. Cell Biol. 99: 615-627.

Huang. N. W. (1973) Mobility and diffusion in the plane of cell membrane. J. Theor. Biol. 40: 11-16.

Jaffe, L. F. (1977) Electrophoresis along cell membranes. Nature 265: $600-602$.

Kidokoro, Y., M. J. Anderson, and R. Gruener (1980) Changes in synaptic potential properties during acetylcholine receptor accumulation and ncuronspecific interactions in Xenopus nerve-muscle cultures. Dev. Biol. 78: 464-483.

Kuromi, H., and Y. Kidokoro (1984) Nerve disperses preexisting acetylcholine receptor clusters prior to induction of receptor accumulation in Xenopus muscle cultures. Dev. Biol. 103: 53-61.

Kuromi, H., B. Brass, and Y. Kidokoro (1985) Formation of acetylcholine receptor clusters at neuromuscular junction in Xenopus cultures. Dev. Biol. 109: 165-176.

Lindstrom, J., W. Gullick, B. Conti-Tronconi, and M. Ellisman (1980) Proteolytic nicking of the acetylcholine receptor. Biochemistry 19 : $4791-4795$.

Luther, P. W., and H. B. Peng (1985) Membrane-related specializations associated with acetylcholine receptor aggregates induced by electric fields. J. Cell Biol. 100: 235-244.

McLaughlin, S., and M.-M. Poo (1981) The role of electro-osmosis in the electric-field-induced movement of charge macromolecules on the surfaces of cells. Biophys. J. 34: 85-93. 
Nieuwkoop, P. D., and J. Faber (1962) Normal Table of Xenopus laevis, North Holland, Amsterdam.

Orida N., and M.-M. Poo (1978) Electrophoretic movement and localization of acetylcholine receptors in the embryonic muscle cell membrane. Nature 275: 31-35.

Orida, N., and M.-M. Poo (1980) On the developmental regulation of acetylcholine receptor mobility in the Xenopus embryonic muscle membrane. Exp. Cell Res. 130: 281-290.

Orida, N., and M.-M. Poo (1981) Maintenance and dissolution of acetylcholine receptor clusters in the embryonic muscle cell membrane. Dev. Brain Res. 1: 293-298.

Peng, H. B., P.-C. Cheng, and P. W. Luther (1981) Formation of ACh receptor clusters induced by positively charged latex beads. Nature (Lond.) 292: 831-834.

Podleski, T. R., and M. M. Salpeter (1988) Acetylcholine receptor clustering and triton solubility: Neural effect. J. Neurobiol. 19: 167185.

Poo, M.-M. (1982) Rapid lateral diffusion of functional ACh receptors in embryonic muscle cell membrane. Nature 295: 332-334.

Poo, M.-M., and K. R. Robinson (1977) Electrophoresis of concanavalin A receptors along embryonic muscle cell membrane. Nature 265: 602-605.

Poo, M.-M., W-J. H. Poo, and J. W. Lam (1978) Lateral electrophoresis and diffusion of concanavalin A receptors in the membrane of embryonic muscle cell. J. Cell Biol. 76: 483-501.

Poo, M.-M., J. W. Lam, N. Orida, and A. W. Chao (1979) Electrophoresis and diffusion in the plane of the cell membrane. Biophys. J. 26: $1-22$.

Pumplin, D. W., and R. J. Bloch (1987) Disruption and reformation of the acetylcholine receptor clusters of cultured rat myotubes occur in two distinct stages. J. Cell Biol. 104: 97-108.
Role, L. W., V. R. Matossian, R. J. O'Brien, and G. D. Fischbach (1985) On the mechanism of acetylcholine receptor accumulation at newly formed synapses on chick myotubes. J. Neurosci. 5: 2197-2204.

Role, L. W., D. Roufa, and G. D. Fischbach (1987) The distribution of acetylcholine receptor clusters and sites of transmitter release along chick ciliary ganglion neurite-myotube contacts in culture. J. Cell Biol. 104: 371-379.

Ryan, T. A., J. Myers, D. Holowka, B. Baird, and W. W. Webb (1988) Molecular crowding on the cell surface. Science 239:61-64.

Scalettar, B. A., J. R. Abney, and J. C. Owicki (1988) Theoretical comparison of the self diffusion and mutual diffusion of interacting membrane proteins. Proc. Natl. Acad. Sci. USA 85: 6726-6730.

Schuetze, S. M., and L. W. Role (1987) Developmental regulation of nicotinic acetylcholine receptors. Annu. Rev. Neurosci. 10:403-457.

Stollberg, J., and S. E. Fraser (1988) Acetylcholine receptors and concanavalin A-binding sites on cultured Xenopus muscle cells: Electrophoresis, diffusion, and aggregation. J. Cell Biol. 107: 1397-1408.

Taylor, R. B., P. H. Duffus, M. C. Raff, and S. de Petris (1971) Redistribution and pinocytosis of lymphocyte surface immunoglobulin molecules induced by anti-immunoglobulin antibody. Nature (Lond.) New Biol. 237: 225-229.

Usdin, T. B., and G. D. Fischbach (1986) Purification and characterization of a polypeptide from chick brain that promotes the accumulation of acetylcholine receptors in chick myotubes. J. Cell Biol. 103: 493-507.

Young, S. H., and M.-M. Poo (1983) Rapid lateral diffusion of extrajunctional acetylcholine receptors in the developing muscle membrane of Xenopus tadpole. J. Neurosci. 3: 225-231.

Ziskind-Conhaim, L., I. Geffen, and Z. W. Hall (1984) Redistribution of acetylcholine receptors on developing rat myotubes. J. Neurosci. 4: $2346-2349$. 